\title{
Efficacy of Low-Level Laser on Renal Pruritus in Hemodialysis Patients Zakaria Mowafy Emam ${ }^{1}$, Sami Ahmed Abbas², Ahmed Hassan El-Thakaby ${ }^{2}$, Marwa Mahdy Abd EL-Hameed ${ }^{1}$, Amany Ibrahim Marae Farek ${ }^{1}$ \\ ${ }^{1}$ Department of Physical Therapy for Surgery, ${ }^{2}$ National Institute of Urology and Nephrology, Faculty of Physical Therapy, Cairo University, Giza, Egypt \\ *Corresponding author: Amany Ibrahim Marae Farek, Mobile: (+20)1012783532, \\ E-Mail: amanyibrahim1234@icould.com
}

\begin{abstract}
Background: Renal pruritus is a distressing disease, often overlooked condition in patients with chronic kidney disease and end-stage renal disease that adversely affect the quality of life (QOL) and medical outcomes of patients and therapy with antihistamines has been unsuccessful.

Objective: The purpose of the study was to evaluate the efficacy of low-level laser on renal pruritus in hemodialysis patients.

Patients and methods: Thirty patients who established diagnosis of chronic renal failure with age ranged 35-60 years were selected randomly from Hemodialysis Unit of Al-Galaa military Hospital. Patients were randomized into two groups of equal number, 15 each. Group (A) received low level laser therapy 3times/week for 8 consecutive weeks and antihistamine and their conventional medical care. Group (B) who only received antihistamine and their conventional medical care.

Parameters: Itching visual analogue scale (VAS) and 5-D Pruritus scale.

Results: Compared to the results before and after low level laser (LLLT) treatment. Our study showed that there was a statistically significant decrease in the results of VAS and 5-D pruritus; but the results of group (A) were superior to that of group (B) when comparing the group results together.

Conclusion: It can be concluded that low level laser therapy (LLLT) relieves itching pain and lowers progression of renal pruritus in patients undergoing hemodialysis.
\end{abstract}

Keywords: Hemodialysis, Renal Pruritus, low level laser therapy, Antihistamine, Itching Visual analogue scale.

\section{INTRODUCTION}

Chronic kidney disease (CKD) is defined as either kidney damage or decreased kidney function (decreased GFR) for 3 or more months (1). Renal pruritus, also known as uremic pruritus (UP), afflicts from $25 \%$ to $86 \%$ of patients with $\mathrm{CKD}^{(2)}$. Currently, antihistamines are prescribed as a standard routine therapy for pruritus, but they never provide full relief on their own for patients with moderate to severe itch. Such patients produce excessive histamine, and antihistamines do not act by desensitizing or inhibiting the itch receptors, so sufficient relief from itching is not attained ${ }^{(3)}$.

It has been suggested that phototherapy is a safe and efficacious management modality which decreases pruritus and can be used across age groups that provide relief for UP patients without many of the risks and adverse effects of systemic medications and probably lowers inflammation and improve quality of life ${ }^{(4)}$. Low level laser therapy (LLLT), phototherapy or photo biomodulation refers to the use of photons at a nonthermal irradiance to alter biological activity. LLLT uses coherent light sources, non-coherent light sources consisting of filtered lamps or LED, or, on occasion, a combination of both. LLLT is a safe and cost-effective treatment modality. The main medical applications of LLLT are reducing pain and inflammation, augmenting tissue repair and promoting regeneration of different tissues and nerves and preventing tissue damage in situations where it is likely to occur ${ }^{(5)}$. Laser treatment inhibits the release of cyclooxygenase, prostaglandins, and cytokine levels, and it accelerates collagen synthesis and cell proliferation. It also decreases pain levels, improves tissue repair, and inhibits $\mathrm{A} \delta$ and $\mathrm{C}$ fiber transmission due to its effect on nerve structures and functional disability ${ }^{(6)}$.

The aim of this work was to evaluate the efficacy of low-level laser on renal pruritus in hemodialysis patients.

\section{PATIENTS AND METHODS}

Thirty men patients who established diagnosis of chronic renal failure (CRF) with age ranged 30-60 years were selected randomly from Hemodialysis Unit of AlGalaa military Hospital, Cairo, Egypt.

The included subjects were randomly distributed into two equal groups; Group A (Study group) consisted of 15 men patients on hemodialysis treatment and received low level laser therapy (LLLT), for 10-15 minutes, 2 times/week, for 2 months in addition to conventional medical treatment (antihistamine), and Group B (Control group) consisted of 15 men patients who only received antihistamine and their conventional medical care. 


\section{Ethical approval:}

Research Ethics Committee and quality control approvals were obtained from the ethical board of Cairo university. The study purpose and procedures were explained in detail and in plain terms to each subject before being asked to give an informed written consent to participate in the study. Quality control of screening, handling of data and verification of adherence to protocols were done on a regular basis by the trial coordinator.

\section{Criteria for the patient selection:}

a- Inclusion Criteria:

- Ages of patients were ranged between 30 and 60 years.

- Men patients diagnosed as CRF.

- All patients were stable on dialysis minimally three months.

- All patients enrolled to the study signed the informed consent.

- All patients were examined medically and referred by nephrologists.

\section{b- Exclusion Criteria:}

The study excluded patients with any of the following:

- Unstable blood pressure.

- Congestive heart failure.

- Hyperkalemia $>6 \mathrm{mmol} / \mathrm{l}$.

- Hypokalemia $<3.5 \mathrm{mmol} / \mathrm{l}$.

- $\quad$ Skin diseases

- Skin pigmentation

- Osteoporosis.

- Anemia.

- Mentally disturbed

- Sensitivity to phototherapy

\section{Procedures of the study:}

The procedure of this study was divided into two main procedures.

\section{A) Measurement procedures:}

Evaluation of efficacy of treatment by Itching visual analogue scale (VAS) and Pruritus 5-D scale.

Visual Analogue Scale (VAS) seems to be one of the most commonly used methods of pruritus severity assessment, as it provides an easy and rapid estimation of itch ${ }^{(7)}$ while the 5-D Itch scale is a questionnaire consisting of 5 items used specifically to measure the course of itch by asking for the degree, duration, disability and distribution of the pruritus within the last 2 weeks ${ }^{(8)}$.

\section{Measurements have been taken as following:}

- Before starting any protocol (pretreatment).

- After two months(8weeks) (post-treatment).

\section{B) Therapeutic procedures:}

Gallium Arsenide (GaAs) diode red model level laser M300 made in Italy was applied perpendicular $\left(90^{\circ}\right)$ on patient affected area while patients were per skin and in prone position. It was a continuous beam laser with an energy density of 4 $\mathrm{J} / \mathrm{cm}^{2}$, wavelength $650 \mathrm{~nm}$, frequency $10 \mathrm{kHz}$ and output power $100 \mathrm{~mW}$. machine delivers laser in scanning mode laser of $60 \mathrm{~cm}$ distance between laser source and wound. All areas were treated 3 times per week for 8 weeks. Each affected are was given laser therapy for duration of 125 second every time ${ }^{(9)}$. Use of internal or external medicines were continued without change before and after LLLT ${ }^{(\mathbf{1 0})}$.

\section{Statistical analysis}

Descriptive statistics and Unpaired t-test were conducted for comparison of subject characteristics between both groups. Normal distribution of data was checked using the Shapiro-Wilk test. Levene's test for homogeneity of variances was conducted to ensure the homogeneity between groups. Unpaired t-test was conducted to compare the mean values of VAS and 5D Pruritus Scale between the group A and B. Paired ttest was conducted for comparison between pre and post treatment in each group. The level of significance for all statistical tests was set at $\mathrm{p}<0.05$. All statistical analysis was conducted through the statistical package for social studies (SPSS) version 25 for windows (IBM SPSS, Chicago, IL, USA) ${ }^{(11)}$.

\section{RESULTS}

\section{Subject characteristics:}

Table 1 showed the mean \pm SD of subjects age of group A and B. There was no significant difference between both groups in the mean age $(p=0.47)$.

Table (1): Basic characteristics of participants:

\begin{tabular}{|c|c|c|c|c|}
\hline & Group A & Group B & & \\
\cline { 2 - 5 } & $\overline{\mathrm{x}} \pm \mathrm{SD}$ & $\overline{\mathrm{x}} \pm \mathrm{SD}$ & MD & p-value \\
\hline \multirow{2}{*}{ Age (years) } & $48.86 \pm 8.2$ & $50.06 \pm 7.81$ & -1.2 & 0.47 \\
\hline
\end{tabular}

$\overline{\mathrm{x}}$, mean; SD, Standard deviation; MD, Mean difference; $p$ value, Probability value 
Effect of treatment on VAS and 5-D Pruritus Scale:

- Within group comparison:

There was a significant decrease in VAS and 5D Pruritus Scale post treatment in the group A and B compared with that pretreatment $(p>0.05)$. The percent of decrease in VAS and 5-D Pruritus Scale in the group A was 38.66 and $40.47 \%$ respectively; and that in group B were 5.04, and $9.33 \%$ respectively (Table 2, Figure 1).

\section{- Between groups comparison:}

There was no significant difference in VAS and 5-D Pruritus Scale between both groups pretreatment $(\mathrm{p}>0.05)$. Comparison between the group $A$ and $B$ post treatment revealed a significant decrease in VAS and 5-D Pruritus Scale of the group A compared with that of the group B (p > 0.0001) (Table 2, Figure 1).

Table (2): Mean VAS and 5-D Pruritus Scale pre and post treatment of group A and B:

\begin{tabular}{|c|c|c|r|r|r|}
\hline & Group A & Group B & & \\
& $\overline{\mathbf{x}} \pm$ SD & $\overline{\mathbf{x}} \pm$ SD & MD & t- valu & p valu \\
\hline VAS & & & & & \\
\hline Pretreatment & $9.13 \pm 0.74$ & $9.33 \pm 0.48$ & -0.2 & -0.87 & 0.39 \\
\hline Posttreatment & $5.6 \pm 0.91$ & $8.86 \pm 0.64$ & -3.26 & -11.37 & 0.0001 \\
\hline MD & 3.53 & 0.47 & & & \\
\hline Percentage of chang & $38.66 \%$ & $5.04 \%$ & & & \\
\hline t- value & 13.81 & 2.82 & & & \\
\hline 5-D Pruritus Scale & $\boldsymbol{p}=\mathbf{0 . 0 0 0 1 * *}$ & $\boldsymbol{p}=\mathbf{0 . 0 1 * *}$ & & & \\
\hline Pretreatment & $20.93 \pm 0.88$ & $20.8 \pm 1.01$ & 0.13 & 0.38 & 0.7 \\
\hline Posttreatment & $12.46 \pm 1.18$ & $18.86 \pm 0.83$ & -6.4 & -17.08 & 0.0001 \\
\hline MD & 8.47 & 1.94 & & & \\
\hline Percentage of chang & $40.47 \%$ & $9.33 \%$ & & & \\
\hline t- value & 39.32 & 7.79 & & & \\
\hline
\end{tabular}

$\overline{\mathrm{x}}$, Mean; SD, Standard deviation; $\mathrm{p}$ value, Probability value; $* *$, Significant

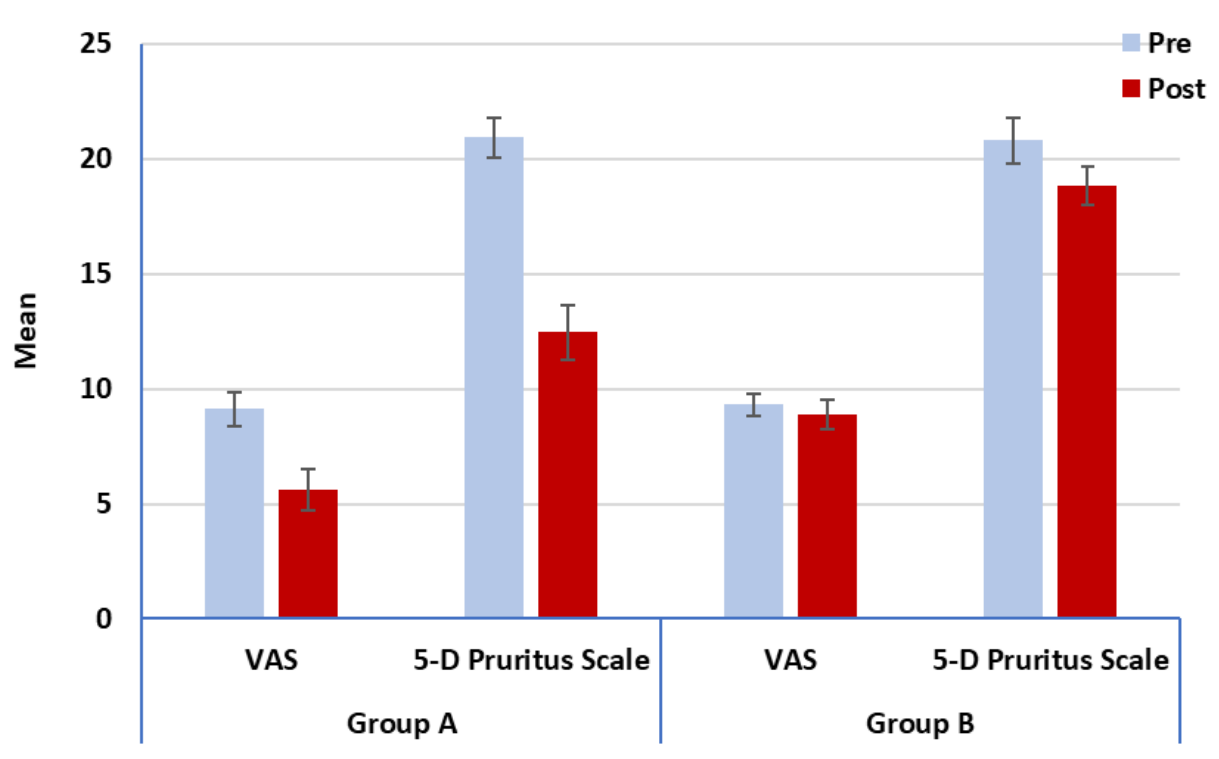

Figure (1): Mean VAS and 5-D Pruritus Scale pre and post treatment of group A and B. 


\section{DISCUSSION}

Pruritus is a common and distressing symptom in patients with CKD. The most recent epidemiologic data have suggested that approximately $40 \%$ of patients with ESRD experience moderate to severe pruritus and that uremic pruritus has a major clinical impact, being associated strongly with poor quality of life, impaired sleep, depression, and increased mortality ${ }^{(\mathbf{1 2})}$.

Currently, antihistamines are prescribed as a standard routine therapy for pruritus, but they never provide full relief on their own for patients with moderate to severe itch. Such patients produce excessive histamine, and antihistamines do not act by desensitizing or inhibiting the itch receptors, so sufficient relief from itching is not attained ${ }^{(3)}$.

Similar to the pain pathway, the pathway for itching sensation engages the spinothalamic tracts and unmyelinated $\mathrm{C}$ fibers and it may be related to descending modulation. Because of the congruity between the itching and pain pathways, it is possible that itching could be susceptible to treatments used for pain control ${ }^{(13)}$. Laser treatment inhibits the release of cyclooxygenase, prostaglandins, and cytokine levels, and it accelerates collagen synthesis and cell proliferation. It also decreases pain levels, improves tissue repair, and inhibits $\mathrm{A} \delta$ and $\mathrm{C}$ fiber transmission due to its effect on nerve structures and functional disability ${ }^{(\boldsymbol{)})}$.

Also, there is no study has reported worsening of kidney function or any negative effects of phototherapy for CKD patients undergoing hemodialysis.

In sum, Phototherapy appears to be safe in this patient population as it does not only slow progression of pruritus, but it could also improve quality of life and survival of HD patients.

The results of the present study showed that there was a significant decrease in VAS and 5-D Pruritus Scale post treatment in the group A and B compared with that pretreatment $(\mathrm{p}>0.05)$. The percent of decrease in VAS and 5-D Pruritus Scale in the group A was 38.66 and $40.47 \%$ respectively; and that in group B were 5.04, and $9.33 \%$ respectively.

Our results agree with the outcomes of Wong $\boldsymbol{e t}$ al. ${ }^{(14)}$ and Simoes et al. ${ }^{(15)}$ studies indicating that low level laser therapy has a better effect on curing disability, relieving pain, and improving QoL according to VAS and QoL questionnaire results.

The finding of our study supported by some previous studies and past literatures that conducted by Brown and Weber ${ }^{(16)}$, Allison et al. ${ }^{(17)}$ and Karu et al. (18) stated that LLLT treatment is non-invasive, painless, very safe, can be easily applied in primary care for a variety of conditions and also is effective for severe pruritus during the process of healing, and the improvements remained at 6 and 12 months after treatment. The symptomatic relief might be due to the beneficial effects of the laser on microcirculation and the pruritogenic chemicals found in tissue and also LLLT stimulates cell proliferation.

Moreover, the results of the present study consistent by the works reported by Hawkins $\boldsymbol{e t}$ al. ${ }^{(19)}$ and Davis ${ }^{(20)}$ state that in the clinical situation, LLLT is an accepted, efficient, noninvasive, and painless method of treating edema, inflammation, stimulate collagen metabolism, wound healing, and promotes fracture healing. Researchers have also found an increase in collagen and elastic fibers in injured tissue and has a photobiomodulation effect over tissue; also, it has photochemical and photomechanical components and no photothermal effect.

Also, Chung et al. (5), Farivar et al. ${ }^{(21)}$ and Ebid and El-Sodany ${ }^{(22)}$ have found on their studied that Low level laser (LLL) is also known as cold laser as it does not produce heating effect, is a safe and costeffective treatment modality that enhances the production and release of endorphins and significantly decreases pain sensation in many conditions, have anti-inflammatory effects and augmenting tissue repair and promoting regeneration of different tissues and nerves, and preventing tissue damage in situations where it is likely to occur.

The study was limited to physical and psychological conditions of the patients that might affect the evaluation and treatment.

\section{CONCLUSION}

Low level laser therapy (LLLT) application for treatment of renal pruritus in HD patients has a significant improvement on their quality of life and relief of the itching pain as evidenced by the highly significant decrease in itching VAS and 5-D Pruritus Scale. The results of the current study would introduce a scientific applicable protocol to help physical therapists, physicians of nephrology and urology in their dealing with HD patients renal pruritus, organize a plan of care to overcome this problem and prevent the progression of it and the development of associated morbidities, including sleep disturbance and depression of patients and to improve the quality of life of HD patients.

\section{Future studies and recommendations:}

The results of this study indicated a need to consider the following recommendations:

1. It was recommended to add low level laser therapy as an integral part of treatment for renal pruritus patients.

2. Further studies are needed to compare between effectiveness of low- intensity laser therapy and high-intensity laser therapy in the treatment of pruritus. 
3. Further studies are needed to compare between effectiveness of low- intensity laser therapy and Broadband Ultraviolet based therapy (BB-UVB) in the treatment of pruritus.

\section{REFERENCES}

1. Remuzzi G, Ruggenenti P, Perico N (2012): Chronic renal diseases: renoprotective benefits of reninangiotensin system inhibition. Ann Intern Med., 13: 410-415.

2. Murphy M, Carmichael A (2000): Renal itch. Clin Exp Dermatol., 25: 103-160

3. Simons F (2004): Advances in $\mathrm{H} 1$-antihistamines. N Engl J Med., 351: 2203-17.

4. Veith W, Deleo V, Silverberg N (2011): Medical phototherapy in childhood skin diseases. Minerva Pediatr., 63: 327-333.

5. Chung H, Dai T, Sharma S et al. (2012): The nuts and bolts of low-level laser (light) therapy." Ann Biomed Eng., 40:516-33.

6. Lim W, Lee S, Kim I et al. (2007): The antiinflammatory mechanism of $635 \mathrm{~nm}$ light emittingdiode irradiation compared with existing COX inhibitors. Lasers Surg Med., 39(7):614-621.

7. Breivik E, Skoglund L (1998): Comparison of present pain intensity assessments on horizontally and vertically oriented visual analogue scales." Methods Find Exp Clin Pharmacol., 20: 719-724.

8. Lai J, Chen H, Chou C et al. (2017): Transformation of 5-D itch scale and numerical rating scale in chronic hemodialysis patients. BMC Nephrol., 18(1):56-59.

9. Gaida K, Koller R, Isler C et al. (2004): Low level laser therapy--a conservative approach to the burn scar? Burns, 30:362-7.

10. Hideki M, Junro K, Mika H et al. (1993): Clinical Application of Low Reactive Level Laser Therapy (LLLT) for Atopic Dermatitis. Keio J Med., 42 (4): 174-176.

11. Winters R, Winters A, Amedee R (2010): Statistics: A brief overview. Ochsner J., 10:213-6.
12. Sara A, Pedro T, Michael J (2015): Pruritus in Kidney Disease. Semin Nephrol., 35(4): 383-391.

13. Wheeler D, Vaux K, Dan T (2000): Use of gabapentin in the treatment of childhood reflex sympathetic dystrophy. Pediatr Neurol., 22(3):220-221.

14. Wong S, Wilder-Smith P (2002): Pilot study of laser effects on oral mucositis in patients receiving chemotherapy. Cancer J., 8(3):247-254.

15. Simoes A, Eduardo F, Luiz A et al. (2009): Laser phototherapy as topical prophylaxis against head and neck cancer radiotherapy-induced oral mucositis: comparison between low and high/lowpower lasers. Lasers Surg Med., 41(4):264-270.

16. Brown A, Weber D (2000): Physical agent modalities. In: Braddom RL (ed) Physical medicine and rehabilitation. WB Saunders, Harcourt Health Sciences Company, London, Pp. 440-458.

17. Allison K, Kiernan M, Waters R et al. (2003): Pulsed dye laser treatment of burn scars. Alleviation or Irritation: Burns, 29: 207-213.

18. Karu T, Pyatibrat L, Kalendo G (2004): Photobiological modulation of cell attachment via cytochromec oxidase. Photochem Photobiol Sci., 3(2):211-216.

19. Hawkins D, Houreld N, Abrahamse H (2005): Low level laser therapy (LLLT) as an effective therapeutic modality for delayed wound healing. Ann N Y Acad Sci., 1056:486-93.

20. Davis C (2009): Complementary Therapies in Rehabilitation: Evidence for Efficacy in Therapy, Prevention, and Wellness. $3^{\text {rd }}$ ed. New Jersey: SLACK Incorporated. Pp. 374-581.

21. Farivar S, Malekshahabi T, Shiari $R$ (2014): Biological effects of low level laser therapy. J Lasers Med Sci., 5:58-62.

22. Ebid A, El-Sodany A (2015): Long-term effect of pulsed high intensity laser therapy in the treatment of post-mastectomy pain syndrome: a double blind, placebo-control, randomized study. Lasers Med Sci., 8:29-35. 